\title{
The effectiveness of virtual reality for people with mild cognitive impairment or dementia: a meta-analysis
}

\author{
Oksoo Kim ${ }^{1}$, Yanghee Pang ${ }^{1}$ and Jung-Hee Kim² ${ }^{*}$ (D)
}

\begin{abstract}
Background: Virtual Reality (VR) is increasingly used in health-related fields and interventions using VR have the potential to be powerful tools in patient management. The aim of this study was to synthesize the effects of VR interventions for people with mild cognitive impairment (MCl) or dementia.

Methods: Electronic databases were searched to identify studies that used an experimental design to investigate VR intervention outcomes for patients with $\mathrm{MCl}$ or dementia. Studies were excluded if the intervention did not focus on VR, if relevant quantitative outcomes were not reported, or if the intended study purpose was assessment or diagnosis. Data were extracted and analyzed from studies that met criteria. To synthesize the intervention effect sizes (ES), we used random effects models to accommodate heterogeneity in the main effect and sub-group analyses. To identify the potential reason for heterogeneity and compare ES according to the moderator variables, subgroup analyses were conducted based on study characteristics and intervention outcomes.

Results: Data from eleven studies that met eligibility criteria were analyzed. VR intervention delivered to participants with $\mathrm{MCl}$ or dementia produced small to medium effects $(E S=0.29, \mathrm{Cl}=0.16,0.42$ ). The $\mathrm{ES}$ for studies using semi-immersive technology ( $E S=0.37, \mathrm{Cl}=0.25,0.49$ ) was greater than the studies using full-immersive VR $(E S=0.03, C l=-0.14,0.21)$. The results showed small-to-medium effects for VR interventions affecting key outcome variables such as cognition ( $E S=0.42, C l=0.24,0.60)$ and physical fitness ( $E S=0.41, C l=0.16,0.65$ ).

Conclusion: VR interventions, particularly of the semi-immersive type, are useful for people with $\mathrm{MCl}$ or dementia. These results should contribute to the establishment of practical guidelines for VR interventions for patients with cognitive decline.
\end{abstract}

Keywords: Mild cognitive impairment, Dementia, Meta-analysis, Virtual reality

\section{Introduction}

Dementia is one of the major causes of disability and dependency among older people. According to the World Health Organization, the number of patients with dementia worldwide is currently estimated at 47 million, and this is expected to increase to 75 million by 2030 and nearly triple by 2050 [1]. The estimated cost of dementia to the global economy increased by $35 \%$ from 2010 to 2015 [2]. Mild cognitive impairment (MCI) is an intermediate stage of cognitive change, between normal

\footnotetext{
* Correspondence: jhee90@catholic.ac.kr

${ }^{2}$ Associate Professor, Department of Clinical Nursing, College of Nursing, The Catholic University of Korea, 222 Banpo-daero, Seocho-gu, Seoul 06591, South Korea

Full list of author information is available at the end of the article
}

aging and dementia; the distinction between $\mathrm{MCI}$ and dementia is determined by the severity of cognitive decline leading to functional impairment [3]. Among elderly Chinese, the prevalence of overall MCI was $20.8 \%$, with higher rates in rural than in urban areas [4]. In a representative Spanish sample, the overall prevalence of MCI was $9.6 \%$, with higher rates in older people and women than in younger people and men [5].

People with cognitive decline report decreased stress when using virtual reality (VR) for both stimulation and relaxation [6]. Patients with cognitive decline may critically benefit from the implementation of VR technology interacting in a multisensory fashion through quasi-naturalistic, realistic stimuli [6-9]. VR interventions have increasingly 
been applied to address phobias, stress, and anxiety in psychotherapy [6] and behavioral therapy [10] as well as in the diagnosis of dementia [11]. VR offers cost-effective, accessible, flexible, and comprehensive interventions for patients who have difficulty attending outpatient appointments due to distance, lack of transport, or disability [6,12]. Given the increasing use of VR in health-related fields, interventions using VR have the potential to be powerful tools in patient management. VR integrates real-time computer graphics, body tracking devices, visual displays, and other sensory inputs, which can be utilized to provide long-term and individualized care for patients with dementia [6]. To our knowledge, guidelines on VR intervention development and the length and dose of effective VR interventions are also lacking. Consequently, this study aimed to synthesize the effects of VR for patients with MCI or dementia.

A systematic review by Coyle et al. [13] examined computerized and VR cognitive training with individuals at high risk for cognitive decline. Further, the effectiveness of multisensory stimulation for individuals with dementia [14] and the use of VR to promote motor recovery in stroke rehabilitation [15] have been reviewed. Other metaanalyses have reviewed the efficacy of VR in examining behavioral assessments [10] and cognitive rehabilitation for brain injuries [16]. However, because the existing literature has mainly focused on the diagnosis of dementia using VR, studies examining the effects of VR intervention are limited and the literature in this area is in its infancy.

A meta-analysis allows researchers to review and assess knowledge in important areas and facilitates evidencebased practice. This statistical technique allows researchers to derive comprehensive results and an objective verification of intervention effectiveness by applying statistical methods to the results of existing scientific studies [17]. By using a meta-analysis, researchers can synthesize findings from studies that are methodologically different to compare the effects of a particular intervention. Important questions in a meta-analysis are whether the methodological, contextual, or substantive differences are related to variation in effect-size parameters.

Virtual environments are defined as "interactive, virtual image displays enhanced by special processing and by non-visual display modalities: to convince users that they are immersed in a synthetic space" [18]. VR is an artificial environment that is created with software and hardware and presented in which users can have closeto-reality experiences [7, 9, 19] VR resembles real-life situations and patients feel the sensation of "presence" or "being there" [7]. Giving immediate performance feedback and offering a personalized environment as well as a higher degree of similarity with the real world could further engage participants [8].

VR systems can be classified into several types according to the virtual environment (e.g., desktop, goggles- and-gloves, large screen, virtual room) and type of interaction technique (e.g., full- immersive, semi-immersive, non-immersive, and passive or active interaction) [6]. VR systems consist of 3D displays that effectively place the patient inside the virtual environment for the highest level of immersion [8]. The simplest form of VR is a 3-D image that can be explored interactively on a personal computer, usually by manipulating keys or the mouse, so that the content of the image moves in some direction or zooms in or out. However, more sophisticated approaches include wrap-around display screens, actual rooms augmented with wearable computers, and haptic devices that let people feel the display images [19].

During VR training, including Wii, participants use wireless controllers to interact with the on-screen avatars via the VR motion detection system. VR training using visual feedback enables participants to see their own movements, a feature that has helped patients adjust their misaligned body center caused by body image damage. In addition, studies show that training serves as a catalyst to active task participation by inducing interest and pleasure and providing immediate visual feedback on performance to enhance motor skills [20,21].

The aim of this study was to synthesize the effects of virtual reality for people with MCI or dementia. The specific research questions considered are as follows:

(a) What is the overall magnitude of the effect of VR for patients with $\mathrm{MCI}$ or dementia?

(b) Which level of dementia stage (MCI, dementia) and intervention setting (community, institution) have the most influence on VR effect size?

(c) Which VR methodology type (game, task) and interaction type (semi-, full-immersive) have the most influence on intervention effect size?

(d) Which study method variables (random allocation) and evaluation methods (self-reported, observer) have the most influence on VR effect size?

\section{Methods}

\section{Data sources and searches}

A systematic search using the following designated keyword combinations were used to search eligible articles: ("dementia" OR "Alzheimer's disease" OR "mild cognitive impairment" OR "cognitive impairment") AND ("virtual reality" or "virtual") without time limit. This study was conducted following the guidelines recommended by the Preferred Reporting Items for Systematic Reviews and Meta-Analyses statement [22]. To identify relevant studies, researchers conducted a systematic search of the following electronic databases: EBSCO, PubMed, ScienceDirect, and comprehensive Korean databases including the Korean Medical Database, Research Information Sharing Service, and National Digital Library. 
The search results were restricted to studies meeting the following inclusion criteria: [1] included a VR intervention; [2] participants had MCI, dementia, or Alzheimer's disease (AD); [3] utilized an experimental design including a control group, case series, randomized or non-randomized design; and [4] were available in full text in English or Korean. Studies were excluded if the intervention did not focus on VR, if relevant quantitative outcomes were not reported, or if the intended purpose was assessment or diagnosis. Further, conference proceedings, case reports, and literature reviews were excluded because they failed to yield effect sizes.

\section{Screening process}

The screening process was conducted by a researcher $(\mathrm{JH})$ and a research assistant (ES). To avoid selection bias, the initial online search was conducted independently by $\mathrm{JH}$ and ES. After exclusion of duplicate studies, titles and abstracts were reviewed; if an abstract was considered relevant or ambiguous, the full text was reviewed jointly, using inclusion and exclusion criteria. Any disagreements in study selection were resolved by discussion until consensus was reached.

The initial electronic database search yielded 768 potentially relevant articles; however, 206 duplicates were excluded. Then, the remaining 562 titles and abstracts were scanned to identify potentially relevant studies. Five-hundred thirty-five did not match the inclusion criteria due to: different target population $(n=91)$, literature reviews $(n=63)$, non-experimental studies $(n=84)$, or irrelevant/non-VR outcomes $(n=297)$. Next, 27 full reports were obtained. Of these, 16 were excluded because they either employed non-experimental studies $(n=5)$ [23-27], presented insufficient results $(n=10)$ [28-36], or reviewed the literature [37]. As a result, data were extracted from a total of 11 studies that met inclusion and exclusion criteria (Fig. 1).

\section{Methodological quality assessment}

We assessed the methodological quality of the included studies using the Risk of Bias Assessment Tool for Nonrandomized Studies (RoBANS), which comprises six domains: participant selection, confounding variables, measurement of exposure, blinding of outcome assessments, incomplete outcome data, and selective outcome reporting [38]. Two authors (OS and $\mathrm{JH}$ ) assessed the RoBANS for all 11 studies and rated eligible studies as having either a high risk, low risk, or uncertain risk of bias. Any disagreements were resolved until consensus was reached. Eight studies were evaluated as high risk for a potential bias in the blinding of outcome assessments. Five studies had a high risk of having confounding variables. Six studies had a risk of exposure measurement bias.

\section{Data extraction}

Data were extracted from studies that met our inclusion criteria by two reviewers (JH and OS) independently. A coding manual including information regarding effect size calculations and study characteristics was developed. The characteristics extracted from each study were: design (year of publication, country of origin, sample size, control group, and evaluation level), patient characteristics (age, dementia stage, and residential status), intervention characteristics (impairment feature, VR environment, VR type, type of interaction technique, session length, and dose), and intervention outcomes. The type of interaction was categorized into three levels of immersion. Interaction with a PC monitor, keyboard, and mouse were non-immersive [39, 40], more sophisticated graphics with larger surface displays were semi-immersive, and 3D displays were coded as fullimmersive (i.e., highest level of immersion) [7, 40]. Intervention outcomes in each study were classified as physical fitness, cognition, emotion, execution, and feasibility. The evaluation of physical fitness included balance ability, gait, and fall measures. Cognition included cognitive ability, memory, concentration, orientation, recall, wording, and attention. Emotion included anxiety, psychological wellbeing, depression, and apathy measures. Verbal response and performance errors were classified in execution. Feasibility usually evaluated barriers to, and facilitators of intervention use, and users' reported experiences with the intervention.

Any discrepancies that arose between the two reviewers were resolved by discussing the coding differences. As a result, no discrepancies remained between researchers for any items in this study, therefore, it was not necessary to calculate the inter-coder reliability.

\section{Data synthesis and statistical analyses}

All effect sizes (ES) were calculated using Comprehensive Meta-Analysis version 2 (Biostat, Englewood, New Jersey). Fixed- and random-effects models address the problem of heterogeneity in distinct ways. A fixedeffects model assumes that primary studies have a common ES. A random-effects model estimates the distribution in the mean ES, assuming that each primary study has a distinct population. We used a random-effects model to accommodate heterogeneity for the main effect and sub-group analyses.

It has been proposed a conditional random effects model in which the choice between models depends on a homogeneity test with the Q-statistic [41]. Significant $\mathrm{Q}$ statistics $(p<.01)$ were identified as heterogeneous. To identify the potential reason for heterogeneity and compare the ES according to the moderator variables, subgroup analyses were conducted based on study characteristics and intervention outcomes. 
Evidence of publication bias was assessed by the "fail-safe N method," which calculates how many missing studies are needed to nullify the effect [42, 43]. Because we needed a large number of studies to nullify the effect, publication bias was not a concern. In this study, the number of missing studies that would bring the $p$-value above .05 was 956 ( $\mathrm{Z}$ value for alpha $=1.959$ ).

To estimate ES of the studies, we used distinct data formats with appropriate formulae: one-group (pretest and posttest) and control group (pretest and posttest). Borenstein et al. [17] commented that systematic reviews could include studies that used independent and matched groups because the ES ( $\mathrm{d}$ or g) has the same statistical meaning regardless of the study design. Therefore, there should be no technical barriers to including studies with different designs in the same analysis.
Utilizing Cohen [44], effect sizes of $0.2,0.5$, and 0.8 were considered small, medium, and large, respectively.

\section{Results}

\section{Study characteristics}

Three of the 11 studies (27.2\%) assessed MCI or questionable dementia, and seven (63.6\%) focused on patients with dementia or AD. Only one study addressed both MCI and dementia. Patients' age range was 63-89 years. Most studies (72.7\%) were conducted in the community; two (18.2\%) were conducted in an institute and one $(9 \%)$ was conducted in both the community and an institute. Interventions were delivered using various VR platforms including a laptop, game controller, VR auditorium, motion sensor, Wii, virtual partner, virtual city, or 3D LCD glasses. Semi-immersive type $(82.8 \%)$ was

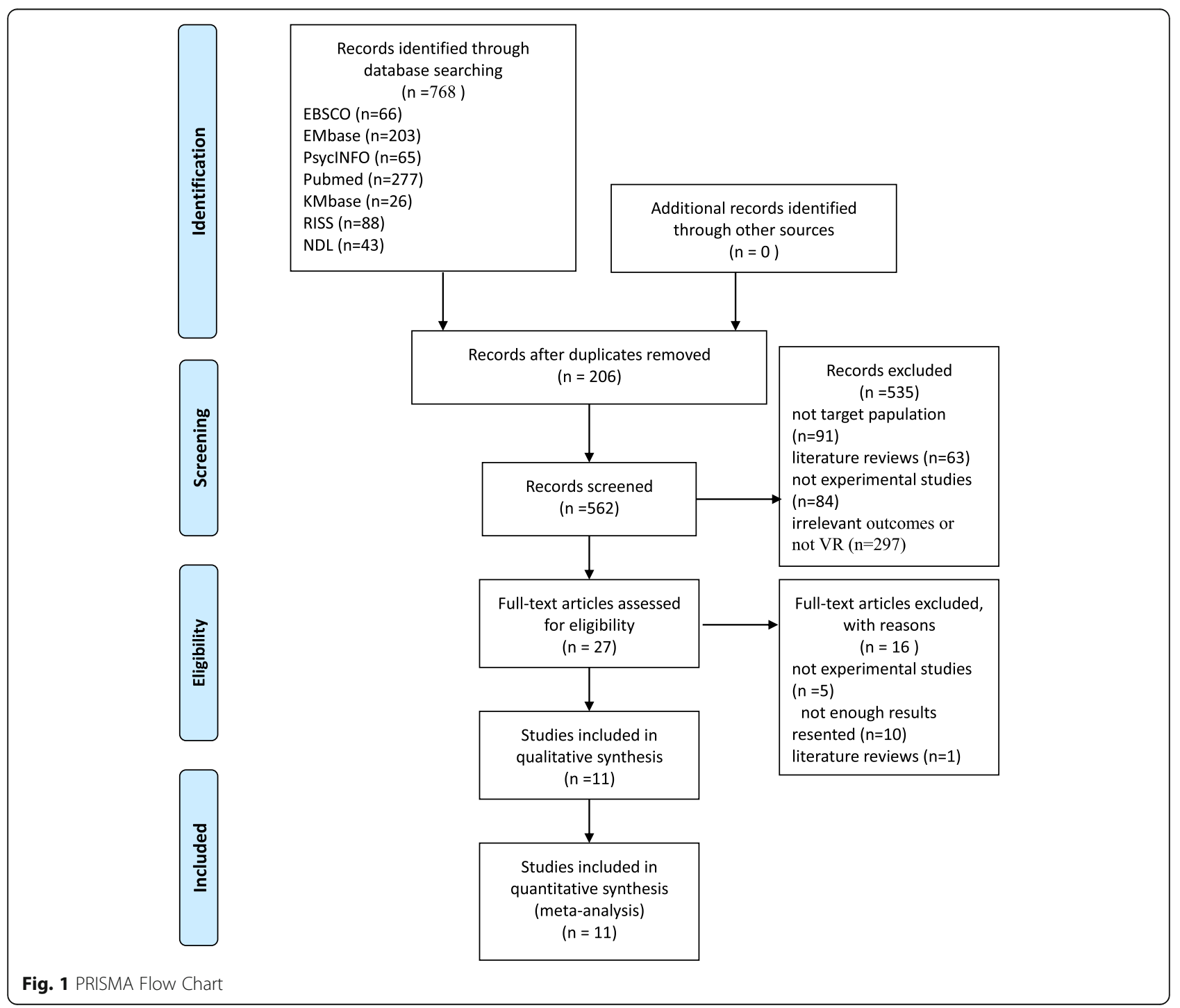




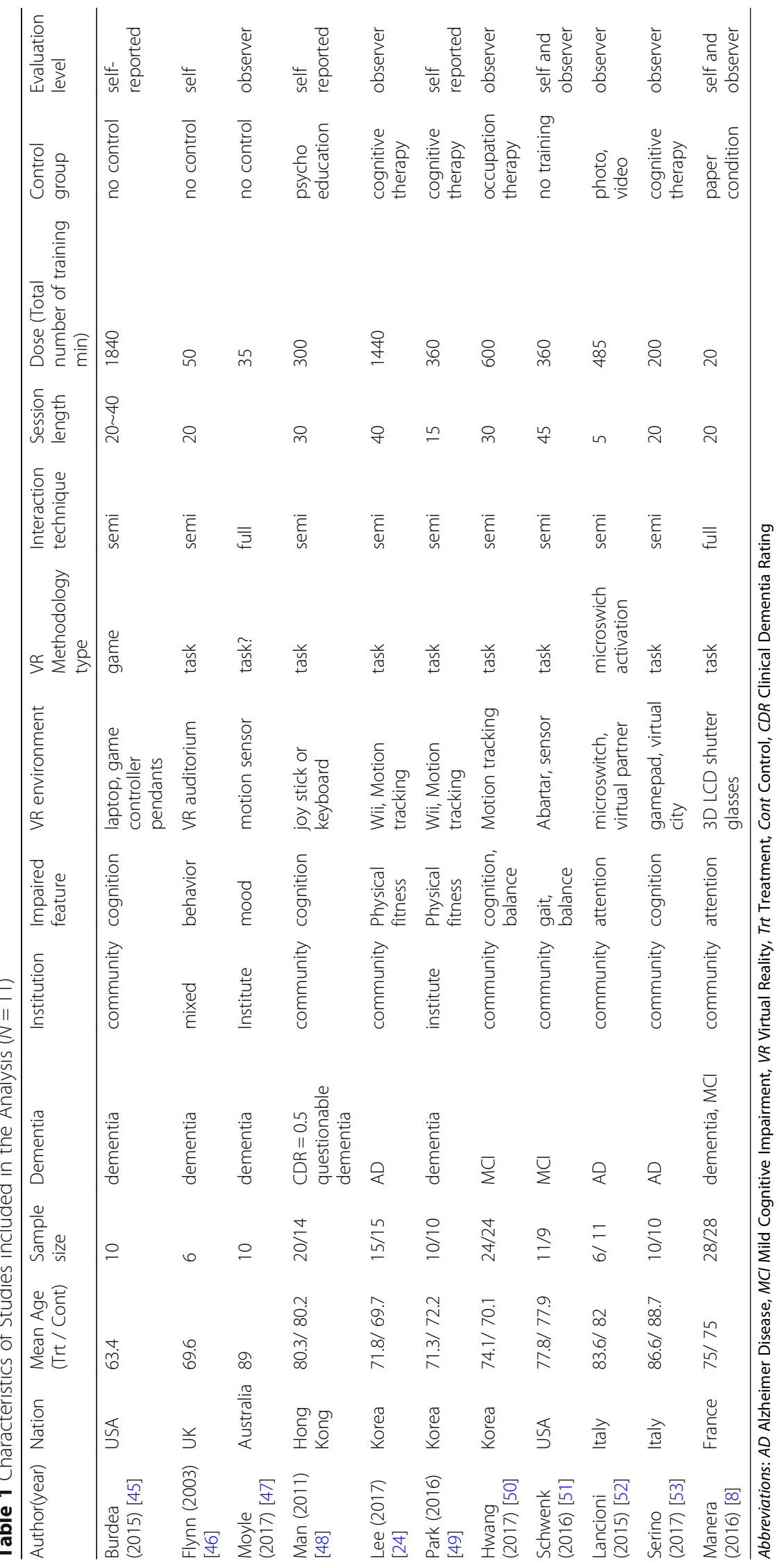


Table 2 Overall Result of the Meta-Analysis using a Random Effects Model

\begin{tabular}{lllcl}
\hline$N$ & $-95 \% \mathrm{Cl}$ & $\mathrm{ES}$ & $+95 \% \mathrm{Cl}$ & $\mathrm{SE}$ \\
\hline 11 & 0.16 & 0.29 & 0.42 & 0.06 \\
\hline
\end{tabular}

Abbreviations: $C l$ confidence interval, $E S$ effect size, $N$ number of studies, $S E$ standard error

the most commonly used and a full-immersive type was applied in two studies (18.2\%; Table 1$)$.

\section{Overall analysis}

When the studies were combined in the meta-analysis, high heterogeneity was observed $(\mathrm{Q}=21.572, p<.001)$. Concerning MCI or AD, VR produced small-to-medium effect sizes using the random-effects model $(E S=0.29$, $\mathrm{CI}=0.16,0.42$ ) (Table 2 and Fig. 2).

\section{Effect sizes per study characteristics}

The meta-analysis provided 70 effect sizes from 11 primary studies. The results of a meta-analysis of the moderators of effects in VR for patients with dementia are shown in Table 3. VR interventions for patients with MCI resulted in greater effects $(E S=0.40, C I=0.23$, $0.58)$ than did interventions for patients with dementia $(\mathrm{ES}=0.35, \mathrm{CI}=0.18,0.51)$, or for the mixed group $(\mathrm{ES}=$ $0.03, \mathrm{CI}=-0.18,0.25)$. Studies conducted in the community $(E S=0.33, C I=0.21,0.45)$ had larger effect sizes than those conducted in an institution $(E S=0.10, C I=-$ $0.25,0.46)$, or in both the community and an institution $(E S=0.07, C I=-0.28,0.43)$. Six studies used two-group posttest, one study used a control group, and four studies used the one-group design. Regarding experimental and control group allocation, random allocation $(\mathrm{ES}=$ $0.36, \mathrm{CI}=0.18,0.53)$ and no randomization $(\mathrm{ES}=0.4$, $\mathrm{CI}=0.19,0.61)$ showed small to moderate effects, which were larger than those with a one-group design (ES = $0.15, \mathrm{CI}=-0.01,0.33)$.

For methodology type, VR using a task $(\mathrm{ES}=0.32, \mathrm{CI}=$ $0.20,0.45)$ resulted in a larger effect than VR using a game $(E S=0.21, C I=0.01,0.42)$. Regarding type of interaction technique, studies using semi-immersive technology $(E S=0.37, C I=0.25,0.49)$ had a greater effect than studies using full-immersive technology $(\mathrm{ES}=0.03, \mathrm{CI}=$ $0.14,0.21$ ). Lastly, regarding type of evaluation, effect sizes were higher for self-reported evaluation $(\mathrm{ES}=0.31$, $\mathrm{CI}=0.10$, 0.52) than for observer-reported evaluation $(\mathrm{ES}=0.30, \mathrm{CI}=0.19,0.42)$.

\section{Effect sizes according to intervention outcomes}

Regarding the VR intervention outcome, the effect size of cognition ( $E S=0.42, C I=0.24,0.60$ ) was higher than that of physical fitness $(E S=0.41, C I=0.16,0.65)$, emotion $(\mathrm{ES}=0.14, \mathrm{CI}=-0.07,0.36)$, execution $(\mathrm{ES}=0.07$,

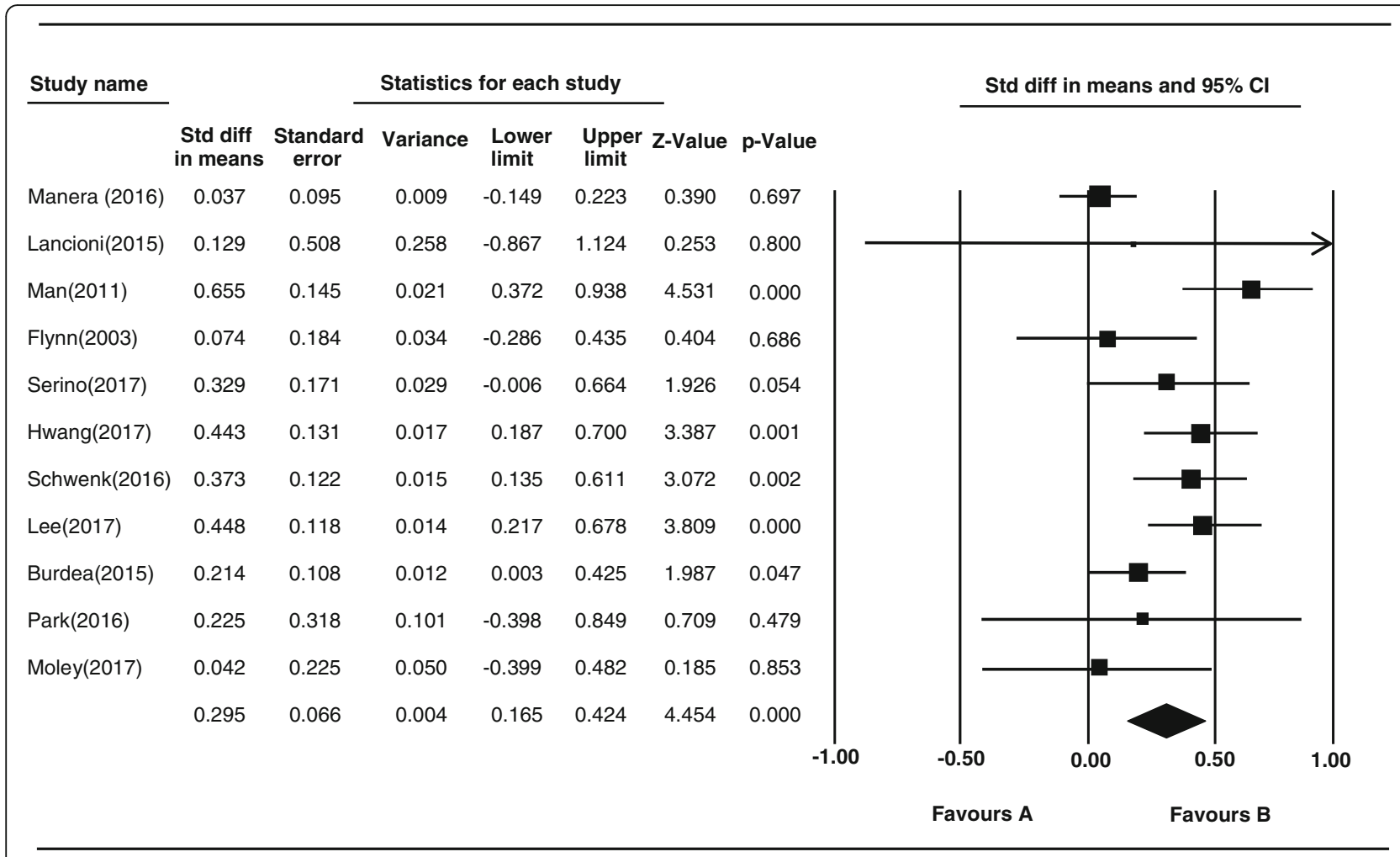

Fig. 2 Forest Plots for Primary Studies 
Table 3 Effect Sizes by Subgroup according to Study Characteristics

\begin{tabular}{|c|c|c|c|c|c|c|c|}
\hline \multirow{2}{*}{$\frac{\text { Subgroup }}{\text { Dementia stage }}$} & \multirow{2}{*}{$\frac{\text { Categories }}{\text { Dementia }}$} & \multirow{2}{*}{$\frac{k}{44}$} & \multirow{2}{*}{$\begin{array}{l}95 \% \mathrm{Cl} \text { ES } \\
0.18\end{array}$} & \multicolumn{2}{|c|}{$+95 \% \mathrm{Cl}$} & \multirow{2}{*}{$\frac{p}{.000}$} & \multirow{2}{*}{$\frac{S E}{0.07}$} \\
\hline & & & & 0.35 & 0.51 & & \\
\hline & $\mathrm{MCl}$ & 18 & 0.23 & 0.40 & 0.58 & .000 & 0.08 \\
\hline & Dementia+ $\mathrm{MCl}$ & 8 & -0.18 & 0.03 & 0.25 & .749 & 0.10 \\
\hline \multirow[t]{3}{*}{ Institution } & Community & 61 & 0.21 & 0.33 & 0.45 & .000 & 0.06 \\
\hline & Mixed & 5 & -0.28 & 0.07 & 0.43 & .686 & 0.14 \\
\hline & Institution & 4 & -0.25 & 0.10 & 0.46 & .575 & 0.18 \\
\hline \multirow[t]{2}{*}{ Methodology type } & Game & 9 & 0.01 & 0.21 & 0.42 & .047 & 0.10 \\
\hline & Task & 63 & 0.20 & 0.32 & 0.45 & .000 & 0.06 \\
\hline \multirow[t]{2}{*}{ Type of interaction technique } & Semi & 60 & 0.25 & 0.37 & 0.49 & .000 & 0.05 \\
\hline & Full & 10 & -0.14 & 0.03 & 0.21 & .684 & 0.09 \\
\hline \multirow[t]{3}{*}{ Randomization } & Yes & 41 & 0.18 & 0.36 & 0.53 & .000 & 0.08 \\
\hline & No & 13 & 0.19 & 0.40 & 0.61 & .000 & 0.44 \\
\hline & NA & 16 & -0.01 & 0.15 & 0.33 & .065 & 0.08 \\
\hline \multirow[t]{2}{*}{ Evaluation } & Self-reported & 29 & 0.10 & 0.31 & 0.52 & .003 & 0.10 \\
\hline & Observer & 38 & 0.19 & 0.30 & 0.42 & .000 & 0.05 \\
\hline
\end{tabular}

Abbreviations: $\mathrm{Cl}$ confidence interval, ES effect size, $k$, number of effect size, SE standard error

$\mathrm{CI}=-0.34,0.49)$ or feasibility $(\mathrm{ES}=0.12, \mathrm{CI}=-0.10,0.34)$ (Table 4).

\section{Discussion}

The aim of this study was to synthesize the effects of 11 experimental studies that used VR interventions with patients with MCI or dementia. The results showed smallto-medium positive effects for VR interventions on key outcome variables such as physical fitness, cognition, and emotion. This indicates that VR interventions can positively affect various clinical outcomes in patients with cognitive impairment, implying that VR interventions improve cognitive and routine functions by stimulating patients' brains $[6,54]$.

In this study, heterogeneity was identified among the individual studies, so a random-effects model was applied. Subgroup analysis was performed per the characteristics of the individual studies. This meta-analysis used a common metric to determine the magnitude of effects in three different data formats using two-group posttest (standardized mean difference), control group (pretest and posttest), and the one-group (pretest and posttest). All intervention effects were calculated and a pooled estimate of the standard deviation for the population was used. As the ES for random allocation (0.36) and no randomization (0.40) among the study with control group were medium, VR interventions were considered useful for people with MCI or dementia.

The VR intervention effects were greater in those examining patients with $\mathrm{MCI}$ as compared to those examining patients with dementia or both dementia and MCI. In addition, the outcome effects were greater when the intervention was conducted in the community as compared to in an institution or both the community and an institution. Consequently, we posit that VR interventions are most effective with patients with $\mathrm{MCI}$ in a community setting. Concerning methodology, a task was more effective than a game. Moreover, semi-immersive technology was more effective than full-immersive technology including 3D display and HMD. Perhaps VR-based intervention programs

Table 4 Effect Sizes by Subgroup according to Intervention Outcomes

\begin{tabular}{|c|c|c|c|c|c|c|c|}
\hline Subgroup & Categories & k & $-95 \% \mathrm{Cl}$ & ES & $+95 \% \mathrm{Cl}$ & $p$ & SE \\
\hline \multirow[t]{5}{*}{ Intervention Outcomes } & Physical fitness & 12 & 0.16 & 0.41 & 0.65 & .001 & 0.12 \\
\hline & Cognition & 36 & 0.24 & 0.42 & 0.60 & .000 & 0.06 \\
\hline & Emotion & 10 & -0.07 & 0.14 & 0.36 & .198 & 0.11 \\
\hline & Execution & 6 & -0.34 & 0.07 & 0.49 & .335 & 0.21 \\
\hline & Feasibility & 7 & -0.10 & 0.12 & 0.34 & .302 & 0.10 \\
\hline
\end{tabular}


provide more diverse, comprehensive, and secure functions but can be complex and difficult for older adults to use [55].

Recently, commercialized VR has developed rapidly and is readily available [6]. However, VR interventions for older adults with reduced vision or other sensory problems may be unsafe [56]. Therefore, it is necessary to consider a methodology type and an interaction technique that will result in the safe implementation of a VR program for people with sensory and cognitive impairment, including older patients with dementia.

The VR intervention effect in the cognition category was small to medium, which is consistent with the results of a systematic review by Coyle et al. [13]. Coyle and colleagues showed that a VR intervention moderately improved the cognitive functioning of participants with cognitive impairment. Further, prior studies showed VR interventions were effective in improving the physical functions and walking speed of community-dwelling patients who had a stroke [54,57]. This suggests that a VR physical fitness intervention may effectively improve the functioning of patients with MCI or dementia.

The intervention effect was slightly higher when the evaluation method was self-reported as compared to observer-reported. For people with cognitive impairment, it is essential to confirm the effectiveness of the program using both subjective evaluations from self- and otherreports [58]. More objective evaluation methods through observation should be implemented in future studies.

VR could also be adapted to patients' needs and characteristics in performing activities, tasks, and tests [7]. $\mathrm{VR}$ is an effective intervention for patients with $\mathrm{AD}$ when it can be performed safely $[6,12]$. Patients with $\mathrm{MCI}$ or dementia reported a VR task to be more satisfying, secure, comfortable, and less anxiety provoking than a paper-based task [8]. Older patients with dementia experience impaired sensory stimulation due to impaired cognitive function and aging, which can result in anxiety, agitated behaviors, and impaired social functioning [14]. In a VR condition, patients experience various forms of sensory stimulation in a comfortable, safe, immersion environment that can promote functional learning as well as the transfer of learned functions [14].

VR interventions positively affected the cognitive and physical functioning of patients with MCI or dementia. However, because most existing studies have focused on the diagnosis of dementia using VR, studies examining the effects of VR intervention are limited. There is also a lack of guidelines on the VR development process and the length and dose of effective interventions. Current study results should contribute to the establishment of practical guidelines for VR intervention for patients with cognitive decline. Because VR is cost-effective, flexible, comprehensive, and useful for patient-centered care, it is likely that the scope of VR activities will grow with further technological innovation [13].

\section{Conclusion}

This meta-analysis integrated individual studies about VR interventions for patients with cognitive impairment because VR is cost-effective, flexible, comprehensive, and potentially useful for patient-centered care. This study showed small-to-medium effects on key outcome variables such as physical fitness, cognition, and emotion. Interventions using VR could be useful for people with $\mathrm{MCI}$ or dementia. These results should contribute to the establishment of practical guidelines for VR intervention for patients with cognitive decline. This study had some limitations. First, most analyzed studies did not produce a significant ES because the sample sizes were small and pilot tests were included. Second, the methodological quality of some studies was low (e.g., not randomizing or blinding assessment outcomes). Despite these limitations, the results presented in meta-analysis suggest a basis for evidence-based interventions for patients with $\mathrm{MCI}$ or dementia. Findings also underscore the need for comprehensive guidelines to develop and implement safe, effective VR interventions to improve functional outcomes for people with cognitive impairment.

\section{Abbreviations \\ AD: Alzheimer Disease; CDR: Clinical Dementia Rating; Cl: Confidence interval; Cont: Control; ES: Effect size; MCl: Mild Cognitive Impairment; $\mathrm{MCl}$ : Mild cognitive impairment; N: Number of studies; RoBANS: Risk of Bias Assessment Tool for Nonrandomized Studies; SE: Standard error; Trt: Treatment; VR: Virtual Reality; VR: Virtual reality}

\section{Acknowledgements}

Not Applicable.

Ethical approval and consent to participate Not applicable.

\section{Authors' contributions}

$\mathrm{JH}$ and OS contributed to conception and design of the study, data collection, conducted the statistical analysis, and interpretation of the data and drafted the manuscript. YH contributed to conduct the interpretation of the data and helped to draft the manuscript. All authors read and approved the final manuscript.

\section{Funding}

This work was supported by a Korea Research Foundation grant funded by the Korean Government [(MOEHRD, Basic Research Promotion Fund) (2017R1E1A1A01075188)]. The funding sources had no involvement in the study design, data collection, analysis and interpretation of data.

\section{Availability of data and materials}

The dataset used and analyzed during the current study is available from the corresponding author on reasonable request.

Consent for publication

Not applicable.

Competing interests

The authors declare that they have no competing interests. 


\section{Author details}

'College of Nursing, Ewha Womans University, 52 Ewhayeodae-gil, Seodaemun-gu, Seoul 03760, South Korea. ${ }^{2}$ Associate Professor, Department of Clinical Nursing, College of Nursing, The Catholic University of Korea, 222 Banpo-daero, Seocho-gu, Seoul 06591, South Korea.

Received: 4 March 2019 Accepted: 12 June 2019

Published online: 12 July 2019

\section{References}

1. WHO. https://www.who.int/news-room/fact-sheets/detail/dementia.

2. Wimo A, Guerchet M, Ali G-C, Wu Y-T, Prina AM, Winblad B, et al. The worldwide costs of dementia 2015 and comparisons with 2010. Alzheimers Dement. 2017;13(1):1-7.

3. Oh E, Lee AY. Mild cognitive impairment. J Korean Neurol Assoc. 2016; 34(3):167-75

4. Jia J, Zhou A, Wei C, Jia X, Wang F, Li F, et al. The prevalence of mild cognitive impairment and its etiological subtypes in elderly Chinese. Alzheimers Dement. 2014;10(4):439-47.

5. Lara E, Koyanagi A, Olaya B, Lobo A, Miret M, Tyrovolas S, et al. Mild cognitive impairment in a Spanish representative sample: prevalence and associated factors. Int J Geriatr Psychiatry. 2016;31(8):858-67.

6. García-Betances Rl, Jiménez-Mixco V, Arredondo MT, Cabrera-Umpiérrez MF. Using virtual reality for cognitive training of the elderly. Am J Alzheimers Dis Other Dement. 2015;30(1):49-54.

7. Baus $\mathrm{O}$, Bouchard $\mathrm{S}$. Moving from virtual reality exposure-based therapy to augmented reality exposure-based therapy: a review. Front Hum Neurosci. 2014:8:112

8. Manera V, Chapoulie E, Bourgeois J, Guerchouche R, David R, Ondrej J, et al. A feasibility study with image-based rendered virtual reality in patients with mild cognitive impairment and dementia. PLoS One. 2016;11(3):e0151487.

9. Flynn S, Palma P, Bender A. Feasibility of using the Sony PlayStation 2 gaming platform for an individual poststroke: a case report. J Neurol Phys Ther. 2007;31(4):180-9.

10. Morina N, ljntema H, Meyerbroker K, Emmelkamp PM. Can virtual reality exposure therapy gains be generalized to real-life? A meta-analysis of studies applying behavioral assessments. Behav Res Ther. 2015;74:18-24.

11. Jekel K, Damian M, Wattmo C, Hausner L, Bullock R, Connelly PJ, et al. Mild cognitive impairment and deficits in instrumental activities of daily living: a systematic review. Alzheimers Res Ther. 2015;7(1):17.

12. Weniger G, Ruhleder M, Lange C, Wolf $\mathrm{S}$, Irle E. Egocentric and allocentric memory as assessed by virtual reality in individuals with amnestic mild cognitive impairment. Neuropsychologia. 2011;49(3):518-27.

13. Coyle H, Traynor V, Solowij N. Computerized and virtual reality cognitive training for individuals at high risk of cognitive decline: systematic review of the literature. Am J Geriatr Psychiatry. 2015;23(4):335-59.

14. Sanchez A, Millan-Calenti JC, Lorenzo-Lopez L, Maseda A. Multisensory stimulation for people with dementia: a review of the literature. Am J Alzheimers Dis Other Dement. 2013;28(1):7-14.

15. Henderson A, Korner-Bitensky N, Levin M. Virtual reality in stroke rehabilitation: a systematic review of its effectiveness for upper limb moto recovery. Top Stroke Rehabil. 2007;14(2):52-61.

16. Shin H, Kim K. Virtual reality for cognitive rehabilitation after brain injury: a systematic review. J Phys Ther Sci. 2015;27(9):2999-3002.

17. Borenstein $M$, Hedges LV, Higgins JP, Rothstein HR. Introduction to metaanalysis: Wiley; 2009.

18. Ellis SR. What are virtual environments? IEEE Comput Graph Appl. 1994; 14(1):17-22

19. TechTarget Network. http://whatis.techtarget.com/definition/virtual-reality.

20. Song GB, Park EC. Effect of virtual reality games on stroke patients' balance, gait, depression, and interpersonal relationships. J Phys The Sci. 2015;27(7):2057-60.

21. Bang YS, Son KH, Kim HJ. Effects of virtual reality training using Nintendo Wii and treadmill walking exercise on balance and walking for stroke patients. J Phys Ther Sci. 2016;28(11):3112-5.

22. Moher D, Liberati A, Tetzlaff J, Altman DG. Preferred reporting items for systematic reviews and meta-analyses: the PRISMA statement. Int J Surg 2010;8(5):336-41.

23. Allain P, Foloppe DA, Besnard J, Yamaguchi T, Etcharry-Bouyx F, Le Gall $D$, et al. Detecting everyday action deficits in Alzheimer's disease using a nonimmersive virtual reality kitchen. J Int Neuropsychol Soc. 2014; 20(5):468-77.

24. Lee JY, Kho S, Yoo HB, Park S, Choi JS, Kwon JS, et al. Spatial memory impairments in amnestic mild cognitive impairment in a virtual radial arm maze. Neuropsychiatr Dis Treat. 2014;10:653-60.

25. Sauzeon H, N'Kaoua B, Pala PA, Taillade M, Auriacombe S, Guitton P. Everyday-like memory for objects in ageing and Alzheimer's disease assessed in a visually complex environment: the role of executive functioning and episodic memory. J Neuropsychol. 2016;10(1):33-58.

26. Shelton JT, Lee JH, Scullin MK, Rose NS, Rendell PG, McDaniel MA Improving prospective memory in healthy older adults and individuals with very mild Alzheimer's disease. J Am Geriatr Soc. 2016;64(6):1307-12.

27. Zygouris S, Giakoumis D, Votis K, Doumpoulakis S, Ntovas K, Segkouli S, et al. Can a virtual reality cognitive training application fulfill a dual role? Using the virtual supermarket cognitive training application as a screening tool for mild cognitive impairment. J Alzheimers Dis. 2015;44(4):1333-47.

28. Alm N, Astell AJ, Gowans G, Dye R, Ellis MP, Vaughan P, et al. Engaging multimedia leisure for people with dementia. Gerontechnology. 2009;8(4): 236-46.

29. Boone C, Anderson WS. Virtual taxi drivers: network interactions underlying memory retrieval. Neurosurgery. 2013;73(6):N16-7.

30. Bormans K, Roe K, De Wachter D. Virtual memory palaces to improve quality of life in Alzheimer's disease. Annu Rev Cyberther Telemed. 2016;14:227-32.

31. Boulay M, Benveniste $S$, Boespflug $S$, Jouvelot $P$, Rigaud AS. A pilot usability study of MINWii, a music therapy game for demented patients. Technol Health Care. 2011:19(4):233-46.

32. Cushman LA, Stein K, Duffy CJ. Detecting navigational deficits in cognitive aging and Alzheimer disease using virtual reality. Neurology. 2008;71(12):888-95

33. Gadler E, Grassi A, Riva G. A rehabilitation protocol for empowering spatial orientation in MCl. A pilot study; 2009.

34. McEwen D, Taillon-Hobson A, Bilodeau M, Sveistrup H, Finestone H. Twoweek virtual reality training for dementia: single case feasibility study. J Rehabil Res Dev. 2014:51(7):1069-76.

35. dP. v. Luria and Fozard as founders for creating suitable environments for people with dementia. Gerontechnology. 2010;9(3):380-7.

36. White PJ, Moussavi Z. Neurocognitive treatment for a patient with Alzheimer's disease using a virtual reality navigational environment. J Exp Neurosci. 2016;10:129-35.

37. Ferro KN, Moraes TM, Zaninotto AL, Paiva WS. Neurocognitive treatment using virtual reality. J Exp Neurosci. 2017;11:1179069517717734.

38. Kim SY, Park JE, Lee YJ, Seo HJ, Sheen SS, Hahn S, et al. Testing a tool for assessing the risk of bias for nonrandomized studies showed moderate reliability and promising validity. J Clin Epidemiol. 2013;66(4):408-14

39. Ma $\mathrm{M}$, Zheng $\mathrm{H}$. Virtual reality and serious games in healthcare. In: Advanced Computational Intelligence Paradigms in Healthcare 6 Virtual Reality in Psychotherapy, Rehabilitation, and Assessment: Springer; 2011. p. 169-92.

40. Garcia-Betances RI, Arredondo Waldmeyer MT, Fico G, Cabrera-Umpierrez MF. A succinct overview of virtual reality technology use in Alzheimer's disease. Front Aging Neurosci. 2015:7:80.

41. Higgins JP, Thompson SG, Deeks JJ, Altman DG. Measuring inconsistency in meta-analyses. Bmj. 2003:327(7414):557-60.

42. Cooper H, Hedges LV, Valentine JC. The handbook of research synthesis and meta-analysis: Russell Sage Foundation; 2009.

43. Rosenthal R. The file drawer problem and tolerance for null results. Psychol Bull. 1979;86(3):638.

44. Cohen J. Statistical power analysis for the behavioral sciences. 1988. Report No.: 0805802835.

45. Burdea G, Polistico K, Krishnamoorthy A, House G, Rethage D, Hundal J, et al. Feasibility study of the BrightBrainer integrative cognitive rehabilitation system for elderly with dementia.Disabil Rehabil Assist Technol. 2015;10(5):421-32.

46. Flynn D, van Schaik P, Blackman T, Femcott C, Hobbs B, Calderon C. Developing a virtual reality-based methodology for people with dementia: a feasibility study. Cyberpsychol Behav. 2003;6(6):591-611.

47. Moyle W, Jones C, Dwan T, Petrovich T. Effectiveness of a Virtual Reality Forest on People With Dementia: A Mixed Methods Pilot Study. Gerontologist. 2017:58(3):478-487.

48. Man DW, Chung JC, Lee GY. Evaluation of a virtual reality-based memory training programme for Hong Kong Chinese older adults with questionable dementia: a pilot study. Int J Geriatr Psychiatry. 2012;27(5):513-20. 
49. Park SH, Shin Jl. The effect of virtual reality using a therapeutic program on depression and the quality of life in dementia.Coach Develop. 2017;18(1);13-9.

50. Hwang J, Lee $\mathrm{S}$. The effect of virtual reality program on the cognitive function and balance of the people with mild cognitive impairment. J Phys Ther Sci. 2017;29(8):1283-6.

51. Schwenk M, Sabbagh M, Lin I, Morgan P, Grewal GS, Mohler J, et al. Sensorbased balance training with motion feedback in people with mild cognitive impairment. J Rehabil Res Dev. 2016;53(6):945-58.

52. Lancioni GE, Singh NN, O'Reilly MF, Sigafoos J, D'Amico F, Ferlisi G, et al. Patients with moderate Alzheimer's disease engage in verbal reminiscence with the support of a computer-aided program: A pilot study. Frontiers in Aging Neuroscience. 2015;7:109.

53. Serino S, Pedroli E, Tuena C, De Leo G, Stramba-Badiale M, Goulene K, et al. A novel virtual reality-based training protocol for the enhancement of the "mental frame syncing" in individuals with Alzheimer's disease: A development-of-concept trial. Frontiers in Aging Neuroscience. 2017:9:240.

54. Neri SG, Cardoso JR, Cruz L, Lima RM, de Oliveira RJ, Iversen MD, et al. Do virtual reality games improve mobility skills and balance measurements in community-dwelling older adults? Systematic review and meta-analysis. Clin Rehabil. 2017;31(10):1292-304.

55. Cherniack EP. Not just fun and games: applications of virtual reality in the identification and rehabilitation of cognitive disorders of the elderly. Disabil Rehabil Assist Technol. 2011;6(4):283-9.

56. Van Schaik P, Martyr A, Blackman T, Robinson J. Involving persons with dementia in the evaluation of outdoor environments. CyberPsychol Behav. 2008;11(4):415-24.

57. Rodriques-Baroni JM, Nascimento LR, Ada L, Teixeira-Salmela LF. Walking training associated with virtual reality-based training increases walking speed of individuals with chronic stroke: systematic review with metaanalysis. Braz J Phys Ther. 2014;18(6):502-12.

58. Huckans M, Hutson L, Twamley E, Jak A, Kaye J, Storzbach D. Efficacy of cognitive rehabilitation therapies for mild cognitive impairment $(\mathrm{MCl})$ in older adults: working toward a theoretical model and evidence-based interventions. Neuropsychol Rev. 2013;23(1):63-80.

\section{Publisher's Note}

Springer Nature remains neutral with regard to jurisdictional claims in published maps and institutional affiliations.

Ready to submit your research? Choose BMC and benefit from:

- fast, convenient online submission

- thorough peer review by experienced researchers in your field

- rapid publication on acceptance

- support for research data, including large and complex data types

- gold Open Access which fosters wider collaboration and increased citations

- maximum visibility for your research: over $100 \mathrm{M}$ website views per year

At $\mathrm{BMC}$, research is always in progress.

Learn more biomedcentral.com/submissions 